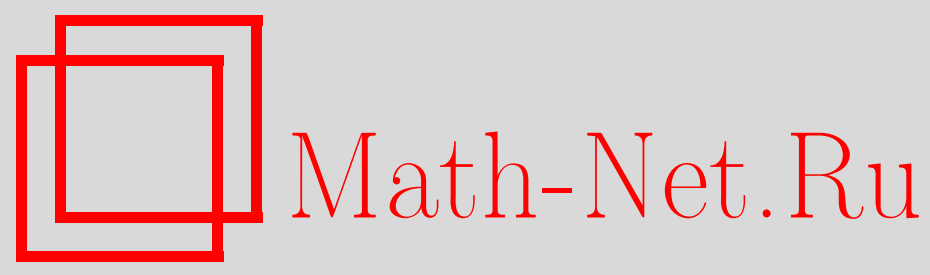

C. А. Терсенов, О задаче Коши для одной системы уравнений ультрапараболического типа, Матем. заметки, 2005, том 77, выпуск 5, 768-774

DOI: https://doi.org/10.4213/mzm2534

Использование Общероссийского математического портала Math-Net.Ru подразумевает, что вы прочитали и согласны с пользовательским соглашением http://www.mathnet.ru/rus/agreement

Параметры загрузки:

IP : 54.198 .55 .26

26 апреля 2023 г., 12:48:10

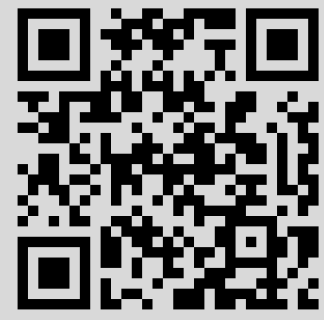




\section{О ЗАДАЧЕ КОШИ ДЛЯ ОДНОЙ СИСТЕМЫ УРАВНЕНИЙ УЛЬТРАПАРАБОЛИЧЕСКОГО ТИПА}

\section{С. А. Терсенов}

Статья посвящена доказательству существования решения задачи Коши для системы уравнений ультрапараболического типа.

Библиография: 13 названий.

Пусть в области $\mathscr{D} \subset \mathbb{R}^{m}$ заданы функции $b_{k}(t) \in C^{1+\alpha}(\mathscr{D}), k=1, \ldots, m, t=$ $\left(t_{1}, \ldots, t_{m}\right), 0<\alpha<1$, и пусть

$$
c_{1}<b_{k}(t)<c_{2} \quad \text { в } t \in \mathscr{D}, \quad k=1, \ldots, m,
$$

где $c_{i}>0$ постоянные. Рассмотрим в области $\mathscr{D}$ гладкую односвязную поверхность $\Gamma$ размерности $m-1$ с параметрическим представлением: $t(\tau)=\left(t_{1}(\tau), \ldots, t_{m}(\tau)\right), \tau=$ $\left(\tau_{1}, \ldots, \tau_{m-1}\right) \in \Gamma_{0}, t(\tau) \in C^{1+\alpha}\left(\Gamma_{0}\right)$. Предположим, что ранг матрицы

$$
Z=\left(\frac{\partial t_{k}(\tau)}{\partial \tau_{i}}\right), \quad k=1, \ldots, m, \quad i=1, \ldots, m-1
$$

равен $m-1$.

В области $Q=\mathbb{R}^{n} \times \mathscr{D}$ рассмотрим систему уравнений ультрапараболического типа

$$
\sum_{|k| \leqslant 2} A_{k}(x, t) D_{x}^{k} u-\sum_{i=1}^{m} b_{i}(t) \frac{\partial u}{\partial t_{i}}=f(x, t),
$$

где $k=\left(k_{1}, \ldots, k_{n}\right),|k|=k_{1}+\cdots+k_{n}, x=\left(x_{1}, \ldots, x_{n}\right) \in \mathbb{R}^{n}, D_{x}^{k}=D_{x_{1}}^{k_{1}} \cdots D_{x_{n}}^{k_{n}}$, $u=\left(u_{1}, \ldots, u_{n}\right), A_{k}$ - матрищы порядка $(N, N)$ с элементами $A_{k}^{i j}, f=\left(f_{1}, \ldots, f_{N}\right)-$ непрерьвная функция в $Q$. В случае $m=1$ считаем, что система (1) параболическая по Петровскому.

В статье рассматривается вопрос о сушествовании решения системы (1) в $Q$, совпадающего с заданной функцией $\phi$ на $\mathbb{R}^{n} \times \Gamma$. Точную постановку задачи и дальнейшие условия на коэффициенты укажем ниже.

Заметим (см. [1]), что при $m \geqslant 2$, если коэффициенты не дифференцируемы по $t$, то дифференцируемых по $t$ решений может не существовать.

Ультрапараболическим уравнениям посвящено много работ. Приведем некоторые из них [1]-[13]. 
В статье будем рассматривать два случая:

1) $\Gamma$ - не характеристическая поверхность уравнения

$$
\sum_{i=1}^{m} b_{i}(t) \frac{\partial w}{\partial t_{i}}=0
$$

2) $Г$-характеристическая поверхность уравнения (2).

1. Поверхность $Г$ не характеристическая. В этом случае определитель

$$
J=\left\|\begin{array}{ccc}
b_{1} & \ldots & b_{m} \\
\partial t_{1} / \partial \tau_{1} & \ldots & \partial t_{m} / \partial \tau_{1} \\
\ldots \ldots \ldots \ldots \ldots \ldots \ldots \ldots \ldots \\
\partial t_{1} / \partial \tau_{m-1} & \ldots & \partial t_{m} / \partial \tau_{m-1}
\end{array}\right\|
$$

не равен нулю на $\Gamma$.

Рассмотрим все характеристические линии (2) проходящие через $\Gamma: t=t(s, \tau) \in$ $C^{1+\alpha}$, которые являются решениями задачи

$$
\frac{d t_{i}}{d s}=b_{i}(t),\left.\quad t_{i}\right|_{s=0}=t_{i}(\tau), \quad i=1, \ldots, m .
$$

Эти линии образуют в $\mathscr{D}$ некоторую область $\mathscr{D}_{0} \subset \mathscr{D}, \Gamma \subset \mathscr{D}$. Пусть $\Lambda(t)-$ решение задачи

$$
\sum_{i=1}^{m} b_{i}(t) \frac{\partial \Lambda}{\partial t_{i}}=1 \quad \text { в } \mathscr{D}_{0},\left.\quad \Lambda\right|_{\Gamma}=0 .
$$

$\Phi$ ункция $\Lambda(t)$ монотонна вдоль характеристических линий. Обозначим через $G_{T} \subset \mathscr{D}_{0}$ множество, где $0<\Lambda(t)<T: G_{T}=\Gamma \times(0<\Lambda(t)<T)$; здесь $T>0$ постоянная.

ЗАДАчА Коши. В области $Q_{T}=\mathbb{R}^{n} \times G_{T}$ ищется ограниченное решение $u=\left(u_{1}\right.$, $\left.\ldots, u_{N}\right)$ системы (1), удовлетворяющее условию

$$
\left.u\right|_{\mathbb{R}^{n} \times \Gamma}=\phi(x, t(\tau)),
$$

где $\phi=\left(\phi_{1}, \ldots, \phi_{N}\right) \in C\left(\mathbb{R}^{n} \times \Gamma\right)$.

1. Рассмотрим систему уравнений

$$
t_{k}(s, \tau)-t_{k}=0, \quad k=1, \ldots, m, \quad(t(s, \tau)-t=0), \quad t \in G_{T} .
$$

Якобиан системы (1.2), равный $J$, не равен нулю на $\Gamma$. Потому при малых $T>0$ система (1.2) однозначно разрешима относительно $s=s(t) \in C^{1+\alpha}\left(G_{T}\right), \tau=\tau(t) \in$ $C^{1+\alpha}\left(G_{T}\right)$, которые являются решениями задач

$$
\sum_{i=1}^{m} b_{i}(t) \frac{\partial s}{\partial t_{i}}=1 \quad \text { в } \quad G_{T},\left.\quad s\right|_{\Gamma}=0, \quad \sum_{i=1}^{m} b_{i}(t) \frac{\partial \tau}{\partial t_{i}}=0 \quad \text { в } \quad G_{T},\left.\quad \tau\right|_{\Gamma}=\left.\tau\right|_{\Gamma_{0}} .
$$


Отсюда заключаем, что $s(t) \equiv \Lambda(t)$.

2. Обозначим через $G_{T}^{(0)}=\Gamma_{0} \times(0<s<T)$ образ $G_{T}$, а через $Q_{T}^{(0)}=\mathbb{R}^{n} \times(0<$ $s<T) \times \Gamma_{0}-$ образ $Q_{T}, B_{\tau}=\mathbb{R}^{n} \times(0<s<T)$.

Пусть $u(x, t)$ - решение задачи Коши. Рассмотрим функцию $u(x, t(s, \tau))=u(x, s ; \tau)$, которая при фиксированном $\tau$ в силу (1.3) будет решением задачи

$$
\begin{gathered}
\sum_{|k| \leqslant 2} A_{k}(x, s ; \tau) D_{x}^{k} u(x, s ; \tau)-\frac{\partial u(x, s ; \tau)}{\partial s}=f(x, s ; \tau) \quad \text { в } \quad B_{\tau}, \\
\left.u\right|_{s=0}=\phi(x, t(\tau)),
\end{gathered}
$$

где $A_{k}(x, s ; \tau)=A_{k}(x, t(s, \tau)), f(x, s ; \tau)=f(x, t(s, \tau))$.

Из единственности решения задачи (1.4), (1.5) следует единственность решения задачи Коши.

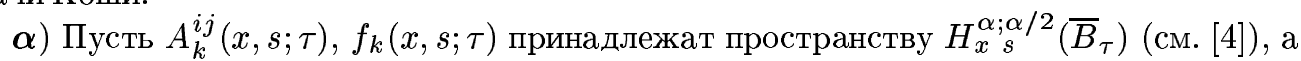
$\phi_{k}$ принадлежит $H_{x}^{2+\alpha}\left(\mathbb{R}^{n}\right)$ и пусть нормы их равномерно относительно $\tau$ ограничены числом $M$. Очевидно, для этого достаточно, чтобы $A_{k}^{i j}(x, t), f_{k}(x, t)$ принадлежали $H_{x t}^{\alpha ; \alpha / 2}\left(\bar{Q}_{T}\right), \phi_{k} \in H_{x}^{2+\alpha}\left(\mathbb{R}^{n}\right)$ (см. [4]).

В силу общей теории при фиксированном $\tau$ имеем.

ЛЕмма 1.1. Существует решение $u(x, s ; \tau)=\left(u_{1}, \ldots, u_{N}\right)$ задачи $(1.4),(1.5)$, әде $u_{k} \in H_{x s}^{2+\alpha ; 1+\alpha / 2}\left(\bar{B}_{\tau}\right) u$

$$
\sum_{k=1}^{N}\left\|u_{k}\right\|_{B_{\tau}}^{(2+\alpha)} \leqslant C \sum_{k=1}^{N}\left(\left\|f_{k}\right\|_{B_{\tau}}^{(\alpha)}+\left\|\phi_{k}\right\|_{\mathbb{R}^{n}}^{(2+\alpha)}\right)
$$

и в силу условий на нормы $f_{k}, \phi_{k}$

$$
\sum_{k=1}^{N} \max _{\tau}\left\|u_{k}\right\|_{B_{\tau}}^{(2+\alpha)} \leqslant C_{1} \sum_{k=1}^{N}\left(\max _{\tau}\left\|f_{k}\right\|_{B_{\tau}}^{(\alpha)}+\max _{\tau}\left\|\phi_{k}\right\|_{\mathbb{R}^{n}}^{(2+\alpha)}\right) \leqslant M_{0} .
$$

3. Рассмотрим $u(x, s ; \tau)$ - решение задачи (1.4), (1.5). При отображении (1.2) $A_{k}(x$, $s(t) ; \tau(t))=A_{k}(x, t), f(x, s(t) ; \tau(t))=f(x, t)$. Если $u(x, s ; \tau)$ непрерьвно дифференцируема по $\tau$, то в силу (1.3) функция $u(x, t)=u(x, s(t) ; \tau(t))$ будет решением задачи Коши в $Q_{T}$. Укажем дополнительные условия, при которых $u(x, s ; \tau)$ непрерьвно дифференцируема по $\tau=\left(\tau_{1}, \ldots, \tau_{m-1}\right)$.

$\boldsymbol{\beta})$ Пусть функции

$$
\frac{\partial A_{k}^{i j}(x, t(s, \tau))}{\partial t_{i}}, \quad \frac{\partial f_{k}(x, t(s, \tau))}{\partial t_{i}}, \quad i=1, \ldots, m
$$

принадлежат $H_{x t}^{\alpha, \alpha / 2}\left(\bar{B}_{T}\right)$ с равномерно относительно $\tau$ ограниченными нормами. Очевидно, для этого достаточно, чтобы

$$
\frac{\partial A_{k}^{i j}(x, t)}{\partial t_{i}} \in H_{x t}^{\alpha, \alpha / 2}\left(\bar{Q}_{T}\right), \quad \frac{\partial f_{k}(x, t)}{\partial t_{i}} \in H_{x t}^{\alpha, \alpha / 2}\left(\bar{Q}_{T}\right), \quad i=1, \ldots, m .
$$


$\Phi$ ункции $\phi_{k}(x, t(\tau))$ непрерьвно дифференцируемы по $t$,

$$
\frac{\partial \phi_{k}(x, t(\tau))}{\partial \tau_{j}}=\sum_{i=1}^{m} \frac{\partial P_{k}}{\partial t_{i}} \frac{\partial t_{i}}{\partial \tau_{j}} \in H_{x}^{2+\alpha}\left(\overline{\mathbb{R}}^{n}\right)
$$

и нормы равномерно ограничены относительно $\tau$. Для этого достаточно, чтобы

$$
\frac{\partial \phi_{k}(x, t(\tau))}{\partial t_{i}} \in H_{x}^{2+\alpha}\left(\overline{\mathbb{R}}^{n}\right)
$$

Функция $\phi_{0}(x, t)=\phi(x, t(\tau(t)))$ есть продолжение $\phi$ в $\mathbb{R}^{n} \times G_{T}$ и в силу $(1.3)$

$$
\sum_{i=1}^{m} b_{i}(t) \frac{\partial \phi_{0}(x, t)}{\partial t_{i}}=0
$$

4. Докажем непрерьвность $u(x, s ; \tau)$ относительно $\tau$. Пусть $u(x, s ; \tau), u\left(x, s ; \tau+\Delta \tau_{i}\right)-$ решения задачи $(1.4),(1.5)$ при $\tau$ и $\tau+\Delta \tau_{i}=\left(\tau_{1}, \ldots, \tau_{i}+\Delta \tau_{i}, \ldots, \tau_{m-1}\right)$. Тогда функция $v(x, s)=u(x, s ; \tau)-u\left(x, s ; \tau+\Delta \tau_{i}\right)$ в $B_{\tau}$ будет решением уравнения

$$
\begin{aligned}
& \sum_{|k| \leqslant 2} A_{k}(x, s ; \tau) D_{x}^{k} v-\frac{\partial v}{\partial s}=f(x, s ; \tau)-f\left(x, s ; \tau+\Delta \tau_{i}\right) \\
& \quad+\sum_{|k| \leqslant 2}\left[A_{k}\left(x, s ; \tau+\Delta \tau_{i}\right)-A_{k}(x, s ; \tau)\right] D_{x}^{k} u\left(x, s ; \tau+\Delta \tau_{i}\right)
\end{aligned}
$$

при начальных условиях

$$
\left.v\right|_{s=0}=\phi(x, t(\tau))-\phi\left(x, t\left(\tau+\Delta \tau_{i}\right)\right) .
$$

В силу условий $(1.6),(1.7)$ и того, что правая часть и начальная функция стремятся к нулю при $\Delta \tau_{i} \rightarrow 0$, функция $v(x, s) \rightarrow 0$, т.е. $u(x, s ; \tau)$ непрерывна относительно $\tau$. Докажем дифференцируемость $u(x, s ; \tau)$ по $\tau$. Пусть $w(x, s ; \tau)$ в $B_{\tau}$ решение уравнения

$$
\sum_{|k| \leqslant 2} A_{k}(x, s ; \tau) D_{x}^{k} w-\frac{\partial w}{\partial s}=\frac{\partial}{\partial \tau_{i}} f(x, s ; \tau)-\sum_{|k| \leqslant 2} \frac{\partial}{\partial \tau_{i}} A_{k}(x, s ; \tau) D_{x}^{k} u(x, s ; \tau),
$$

при начальных условиях

$$
\left.w\right|_{s=0}=\frac{\partial}{\partial \tau_{i}} \phi(x, t(\tau))
$$

В силу условий $\alpha), \beta)$ и (1.7) $w \in H_{x, s}^{2+\alpha, 1+\alpha / 2}\left(\bar{B}_{\tau}\right)$. Рассмотрим разность

$$
W=\frac{u\left(x, s ; \tau+\Delta \tau_{i}\right)-u(x, s ; \tau)}{\Delta \tau_{i}}-w(x, s ; \tau), \quad \Delta \tau_{i} \neq 0,
$$


которая в $B_{\tau}$ является решением уравнения

$$
\begin{aligned}
& \sum_{|k| \leqslant 2} A_{k}(x, s ; \tau) D_{x}^{k} W-\frac{\partial W}{\partial s}=\frac{f\left(x, s ; \tau+\Delta \tau_{i}\right)-f(x, s ; \tau)}{\Delta \tau_{i}}-\frac{\partial}{\partial \tau_{i}} f(x, s ; \tau) \\
& \quad+\sum_{|k| \leqslant 2} \frac{\partial}{\partial \tau_{i}} A_{k}(x, s ; \tau) D_{x}^{k}\left[u(x, s ; \tau)-u\left(x, s ; \tau+\Delta \tau_{i}\right)\right]
\end{aligned}
$$

и удовлетворяет начальному условию

$$
\left.W\right|_{s=0}=\frac{1}{\Delta \tau_{i}}\left[\phi\left(x, \tau+\Delta \tau_{i}\right)-\phi(x, \tau)\right]-\frac{\partial}{\partial \tau_{i}} \phi(x, \tau) .
$$

Как и выше $W \rightarrow 0$, когда $\Delta \tau_{i} \rightarrow 0$,

$$
\frac{\partial}{\partial \tau_{i}} u(x, s ; \tau)=w(x, s ; \tau)
$$

Итак имеем утверждение.

ТЕОРемА 1.1. Пусть коэффиииенты системы (1) и начальные функиии удовлетворяют вышеуказанным условиям и Г - нехарактеристическая поверхность (2). Тогда в $Q_{T}$ существует единственное классическое решение $u \in C_{x, t}^{2,1}\left(Q_{T}\right)$. Функиии $u_{i}(x, s ; \tau)$ в $B_{\tau}$ при произвольном $\tau \in \Gamma_{0}$ принадлежсат $H_{x, t}^{2+\alpha, 1+\alpha / 2}\left(\bar{B}_{\tau}\right) u$ имеют место оценки (1.6), (1.7).

\section{2. Поверхность Г характеристическая.}

ЗАДАчА Коши. В некоторой окрестности $\mathbb{R}^{n} \times G_{T}$ ищется решение $u=\left(u_{1}, \ldots, u_{N}\right)$ системы

$$
\sum_{k=1}^{m} b_{k}(t) \frac{\partial u_{i}}{\partial t_{k}}=\sum_{j=1}^{N} \sum_{|k| \leqslant 2} A_{k}^{i j}(x, t) D_{x}^{k} u_{j}-f_{i}(x, t)
$$

при начальных условиях

$$
u_{i}{\mid \mathbb{R}^{n} \times \Gamma}=\phi_{i}(x, t(\tau)), \quad i=1, \ldots, N
$$

В этом случае определитель $J$ равен нулю на Г. Пусть решение задачи $(2.1),(2.2)$ существует и коэффищиенты удовлетворяют условиям п. 1. При заданном $i$ найдем значение $\partial u_{i} / \partial t_{k}$ на $\mathbb{R}^{n} \times \Gamma$. Из $(2.1),(2.2)$ имеем

$$
\begin{aligned}
\sum_{k=1}^{m} b_{k}(t(\tau)) \frac{\partial u_{i}}{\partial t_{k}} & =\sum_{j=1}^{N} \sum_{|k| \leqslant 2} A_{k}^{i j}(x, t(\tau)) D_{x}^{k} \phi_{j}(x, t(\tau))-f_{i}(x, t(\tau)), \\
\sum_{k=1}^{m} \frac{\partial t_{k}(\tau)}{\partial \tau_{j}} \frac{\partial u_{i}}{\partial t_{k}} & =\frac{\partial}{\partial \tau_{j}} \phi_{i}(x, \tau), \quad j=1, \ldots, m-1 .
\end{aligned}
$$

Определитель системы $(2.3),(2.4)$ относительно $\partial u_{i} / \partial t_{k}, k=1, \ldots, m$, равен определителю $J$, которьй равен нулю на $Г$. Для существования решения системы нужно, чтобы ранг расширенной матрицы был равен рангу основной матрицы, которьй равен $m-1$, 
рангу $Z$. В силу этого нетрудно доказать (как и в случае уравнений с частными производными первого порядка), что существуют функции $\delta_{k}(\tau), k=1, \ldots, m-1$, не зависящие от $i$, такие, что

$$
\sum_{k=1}^{m} \delta_{k}(\tau) \frac{\partial t_{l}(\tau)}{\partial \tau_{k}}=b_{l}(t(\tau)), \quad l=1, \ldots, m
$$

и

$$
\sum_{k=1}^{m} \delta_{k}(\tau) \frac{\partial \phi_{i}(x, t(\tau))}{\partial \tau_{k}}=\sum_{j=1}^{N} \sum_{|k| \leqslant 2} A_{k}^{i j}(x, t(\tau)) D_{x}^{k} \phi_{j}(x, t(\tau))-f_{i}(x, t(\tau))
$$

или

$$
\sum_{k=1}^{m} \delta_{k}(\tau) \frac{\partial \phi(x, t(\tau))}{\partial \tau_{k}}=\sum_{|k| \leqslant 2} A_{k}(x, t(\tau)) D_{x}^{k} \phi(x, t(\tau))-f(x, t(\tau)) .
$$

Рассмотрим на $\Gamma_{0}$ систему уравнений

$$
\frac{d \tau_{k}}{d s}=\delta_{k}(\tau), \quad k=1, \ldots, m-1 .
$$

Через каждую точку $\tau^{0} \in \Gamma_{0}$ проходит одна и только одна линия - решение системы $(2.7)$ $\tau=\tau(s)$, которые покрывают $\Gamma_{0}$. Образы их на $\Gamma: t(s)=t(\tau(s))=\left(t_{1}(s), \ldots, t_{m}(s)\right)$ в силу $(2.5),(2.7)$ удовлетворяют уравнениям

$$
\frac{d t_{i}(s)}{d s}=b_{i}(t(s)), \quad i=1, \ldots, m,
$$

которые и будут характеристическими линиями, покрывающими $Г$. Рассмотрим на $Г$ произвольную фиксированную характеристическую линию $\chi_{s}=\left(t_{1}(s), \ldots, t_{m}(s)\right)$ и пусть $B_{s}=\mathbb{R}^{n} \times \chi_{s}$. В силу (2.6)-(2.8) имеем

$$
\frac{\partial \phi(x, s)}{\partial s}=\sum_{|k| \leqslant 2} A_{k}(x, s) D_{x}^{k} \phi(x, s)-f(x, s),
$$

где $\phi(x, s)=\phi(x, t(\tau(s))), A_{k}(x, s)=A_{k}(x, t(\tau(s))), f(x, s)=f(x, t(\tau(s)))$.

Итак, если $\Gamma$ - характеристическая поверхность, то для разрешимости задачи Коши с данными $\phi$ на $\mathbb{R}^{n} \times \Gamma$ необходимо, чтобы для произвольной характеристической линии $\chi_{s} \subset \Gamma$ след функции $\phi$ на $\mathbb{R}^{n} \times \chi_{s}$ удовлетворял уравнению (2.9). Покажем, что это условие достаточно и решений задачи Коши бесконечно много.

В области $\mathscr{D}$ рассмотрим произвольную нехарактеристическую поверхность $\Gamma^{\prime}$ уравнения (2) размерности $m-1$ с параметрическим представлением $z(\tau)=\left(z_{1}(\tau), \ldots\right.$, $\left.z_{m}(\tau)\right), \tau=\left(\tau_{1}, \ldots, \tau_{m-1}\right) \in \Gamma_{0}^{\prime}, z \in C^{1+\alpha}$, которая пересекает $\Gamma$ по $(m-2)$-мерному многообразию $E$. Характеристические линии, проходящие через $E$, покрывают $\Gamma$. Рассмотрим все характеристические линии уравнения $(2)$, проходящие через $\Gamma^{\prime}$. Они содержат $\Gamma$. Построим область $G_{T}^{\prime}$, аналогичную области $G_{T}$ в п. 1. Пусть $\Gamma_{T}-$ часть $\Gamma$, лежащая в $G_{T}^{\prime}$. Пусть $\phi(x, s)$ - заданная на $\mathbb{R}^{n} \times \Gamma_{T}$ функция, которая при любой характеристической линии $\chi_{s} \in \Gamma_{T}$ является решением системы $(2.9)$ и при $s=0$, т.е. на $\mathbb{R}^{n} \times E$, принимает значения $\phi(x, z(\tau))$. Рассмотрим на $\mathbb{R}^{n} \times \Gamma^{\prime}$ произвольную гладкую функцию $\Psi(x, z(\tau))$, которая совпадает с $\phi(x, z(\tau))$ на $\mathbb{R}^{n} \times E$. В силу теоремы 1.1 существует решение $v=\left(v_{1}, \ldots, v_{N}\right)$ системы $(1)$ в $\mathbb{R}^{n} \times G_{T}^{\prime}$, принимаюшее значение $\Psi(x, z(\tau))$ на $\mathbb{R}^{n} \times \Gamma^{\prime}$. В силу того, что $\Psi=\phi$ на $\mathbb{R}^{n} \times E$, и единственности решения задачи Коши имеем, что $v=\phi(x, s)$ на $\mathbb{R}^{n} \times \Gamma_{T}$. Итак, имеем утверждение. 
ТЕОрема 2.1. Если Г -характеристическая поверхность (2), то для существования решения задачи Коши необходимо и достаточно, чтобь при произвольной характеристической линии $\chi_{s} \subset \Gamma$ след функции фна $\mathbb{R}^{n} \times \chi_{s}$ удовлетворял системе (2.9).

ЗАмечАниЕ. Аналогично может быть исследована задача Коши и задача Дирихле (первая краевая задача) и для системы уравнений:

$$
\sum_{|k| \leqslant 2 p} A_{k}(x, t) D_{x}^{k} u-\sum_{k=1}^{m} b_{k}(t) \frac{\partial u}{\partial t_{k}}=f(x, t), \quad p \geqslant 2
$$

\section{СПИСОК ЦИТИРОВАННОЙ ЛИТЕРАТУРЫ}

[1] Терсенов С. А. Об основных краевых задачах для одного ультрапараболического уравнения // Сиб. матем. ж. 1999. Т. 40. №6. С. 1364-1376.

[2] Генчев Н.С. Об ультрапараболических уравнениях // Докл. АН СССР. 1963. Т. 151. № 2. C. 205-268.

[3] Владимиров В. С., Дрожжинов Ю. Н. Обобщенная задача Коши для ульрапараболического уравнения // Изв. АН СССР. Сер. матем. 1967. Т. 31. № 6. С. 1341-1360.

[4] Ладыженская О. А., Солонников В. А., Уральцева Н. Н. Линейные и квазилинейные уравнения параболического типа. М.: Наука, 1967.

[5] Амиров Ш. Смешанная задача для ультрапараболического уравнения в ограниченных областях // Корректно поставленные краевые задачи для неклассических уравнений математической физики. Новосибирск: ИМ СО АН СССР, 1984.

[6] Гомбоев Л. Г. О корректно поставленной задаче для уравнения ульрапараболического типа // Некоторые проблемы дифференциальных уравнений и дискретной математики. Новосибирск: НГУ, 1986. С. 44-51.

[7] Пятков С. Г. Разрешимость краевых задач для одного ультрапараболического уравнения // Неклассические уравнения и уравнения смешанного типа. Новосибирск: ИМ СО АН СССР, 1990. C. $182-197$.

[8] Шатыро Я.И. Первая краевая задача для одного ультрапараболического уравнения // Дифференц. уравнения. 1971. № 7. С. 1089-1141.

[9] Терсенов А. С. Априорные оценки для одного класса вырождающихся параболических и ульрапараболических уравнений // Докл. РАН. 1994. Т. 338. № 2. С. 168-170.

[10] Manfredini M. The Dirichlet problem for a class of ultraparabolic equations // Adv. Differential Equations. 1997. V. 2. № 5. P. 831-866.

[11] Polidoro S. On a class of ultraparabolic operators of Kolmogorov-Fokker-Planck type // Matematiche (Catania). 1994. V. 49. № 1. P. 53-105.

[12] Ильин А. М. Об одном классе ультрапараболических уравнений // Докл. АН СССР. 1964. T. 159. №6. C. 1214-1217.

[13] Kirov G.Hr. The Dirichlet problem for a certain ultraparabolic equation // Godišnik Visš. Tehn. Učebn. Zaved. Mat. 1970. V. 6. № 2. P. 101-118.

Институт математики им. С. Л. Соболева СО РАН, г. Новосибирск 